\title{
Quantification of Valve Leakage Rates
}

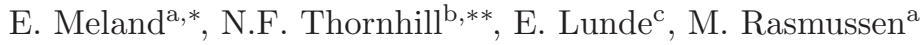 \\ ${ }^{a}$ Marine Systems Research Group, Institute of Marine Technology, Norwegian University of Science and Technology, \\ Trondheim, Norway \\ ${ }^{b}$ Centre for Process Systems Engineering, Department of Chemical Engineering, Imperial College London, UK \\ ${ }^{c}$ Integrated Operations 83 Process Control, Statoil ASA / Dynamica AS, Trondheim, Norway
}

\begin{abstract}
This paper gives an overview of methods concerning the detection and quantification of internal leaks through valves. It also presents comparisons of the methods using new experimental data with emphasis on the spectral information in the signals. The first method utilizes known analytical relationships between acoustic emissions and fluid flow. The second method is a data-driven comparative approach where an on-line signal from a leaking valve is compared to a set of saved reference signals from leaking valves. The work presents a new and improved way of leak estimation compared to what is practiced in the industry today, which will ultimately result in safer operations and reduced maintenance related costs.
\end{abstract}

Keywords: Valve Leak Monitoring, Fault Detection and Diagnosis, Acoustic Emission, Independent Component Analysis, Regression

\section{Introduction}

A shut-off valve has the sole purpose of stopping flow when it is closed. Through leakage refers to a shut off valve in the closed position that fails to stop the flow. The leak can cause a direct financial loss through the loss of a commodity, e.g., hydrocarbon gas, and can be a threat to the environment, equipment and personnel safety. In high risk industry, rigorous time-consuming leakage tests must be conducted to ensure that the functionality of critical valves is adequate.

There has been much research on detection and estimation of through leakage in closed shut down valves. Estimation of leakage rates can in general be done in two ways. The first uses analytical methods based on known physical relationships from which empirical expressions are deduced. These models need empirical inputs, because the problem is too complex to model by pure analytical expressions. The most relevant example of this method was published by Kaewwaewnoi et al. ${ }^{1}$, where the leak rate of two types of valves was determined by a known relationship between acoustic emission and the leak rate. This relationship is examined further in the next section.

The second way of estimating leaks is by direct comparison to other valves that are known to have leaks. The data from these reference leaky valves is collected in a test rig. The comparison method between a new leaky valve and the reference set can be done with, e.g., regression ${ }^{2}$. New data can be collected by manual inspection or by online monitoring. The focus in this paper are methods that can be applied remotely and with minimal intrusion and downtime required to the system. The instrumentation that has been the most thoroughly investigated for automated leak detection includes: Acoustic Emission $(A E)^{3-10}$, vibration monitoring ${ }^{11,12}$ and cavity Dynamic Pressure (DP) monitoring ${ }^{13}$.

\footnotetext{
*Principal Corresponding author

** Corresponding author

Email addresses: erlend.meland@ntnu.no (E. Meland), n.thornhill@imperial.ac.uk (N.F. Thornhill), elunde@statoil.com (E. Lunde), magnus.rasmussen@ntnu.no (M. Rasmussen)
} 
The work in this paper is related to and uses data from a previous study where the frequency spectra of acoustical and dynamic pressure sensors were used to investigate leak signatures on a damaged closed ball valve $^{14}$. The aim was to investigate the potential of frequency spectra to provide signature features. These are otherwise not taken advantage of in single value time variables, e.g., the Root Mean Square (RMS) of the signal. In addition, spectral analysis has several advantages compared to time domain analysis. It lowers the effect of outliers and missing values, and is invariant to phase shifts or time delays.

(Figure 1 about here)

Figure 1 shows a classification of methods in the literature for detection and quantification of valve leakage. They are divided into analytical methods and methods based on comparison. The comparison methods are divided into regression methods and direct spectrum comparisons methods. The regression methods marked with dotted box edges signify non-linear methods. The paper includes a comparative survey of the literature related to the methods in the classification tree. It also provides a comparison of the performance of a selection of them on experimental data from a test rig. The Kaewwaewnoi model and the di-quad model are selected as analytical models. The di-quad model is an original contribution of this paper which is a modification of the Kaewwaewnoi model. The direct spectrum comparison has several types of input features that can be compared. PLSR and KPLSR are selected as the regression methods, and the signal analysis features that are used in the direct spectrum comparison methods are also used in the selected regression methods. Spectral signals in a non-linear model is original work that has never been published before with regards to quantification of valve leakage.

The contribution of the paper is to provide a recommendation, with evidence from analysis of experimental data, of the methods that work best. It also presents a new way of determining valve leakage that has the potential to greatly improve industrial valve leakage detection in oil and gas and other industries.

The paper is divided into sections that follow the classification tree in Figure 1, where dedicated descriptions of the analytical modeling methods, direct spectrum comparative methods, and regression methods can be found. After the descriptions, the following sections are dedicated to performance of the methods with experimental data collected from a test rig.

\section{Analytical and Empirical Modeling}

There have been many attempts at predicting the noise emitted from control valves ${ }^{5,15-26}$. This field of study has similarities to the detection of leaky valves. The objective in these cases has been to determine if the noise levels are low enough to comply with various noise regulations. There have also been attempts at correlating emitted noise to leakage rates. Kaewwaewnoi et al. ${ }^{1}$ attempted to create a model for valve leakage based on control valve noise prediction models. This section introduces the method developed by Kaewwaewnoi et al. and a similar new suggested model.

\section{Kaewwaewnoi's Leakage Modeling Method}

Kaewwaewnoi et al. ${ }^{1}$ use a theoretical model to predict liquid leakage rates through a valve based on acoustical emission. The model is referred to as the Kaewwaewnoi model. The model was reported to be successful based on experiments with ball and globe valves. Leakage was induced by partially opening the valves. The following equation was formed for the valve leakage estimation:

$$
(A E)_{R M S}=C_{1} \frac{1}{\alpha^{5} \rho^{3} D^{14}}\left(\frac{Q}{C_{v}}\right)^{8}\left(\frac{P_{1} S}{\Delta P}\right)^{4}
$$

In the equation, $C_{1}$ is a constant covering fluid variables with some neglected factors, the main ones being: the effect of the AE sensor, valve material and signal attenuation. $A E$ is the measured acoustic emission signal and $R M S$ denotes the root mean square of the signal. $\alpha$ is the sound velocity in the fluid, $\rho$ is fluid density, $D$ is the valve size the, $Q$ is the volume flow rate, $\Delta P$ is pressure drop across the valve, $C_{v}$ is the valve flow coefficient, and $S$ is the specific gravity of the liquid. $C_{v}$ are table values from ${ }^{27}$. $C_{1}$ is determined experimentally and Equation 1 is then solved for $Q$. 


\section{Di-quad Valve Noise Modeling Method}

Kaewwaewnoi et al. uses a quadrupole relationship (a quadrupole refers to a geometrical arrangement of emission sources) to describe leakage. El-Shorbagy ${ }^{5}$ explored a similar model. Instead of having just a quadrupole, a dipole was also added for modeling control valve noise. The dipole source was added because quadrupole and dipole terms are the prominent noise sources present within the turbulent field of a jet flow ${ }^{20}$. The dipole addition follows from Curle's theory ${ }^{28}$. Adding a dipole source to Kaewwaewnoi's equation (Equation 1) yields the following equation:

$$
(A E)_{R M S}=\frac{\left(C_{1} P_{1}+C_{2}\right) P_{1}^{3}}{\alpha^{5} \rho^{3} D^{14}}\left(\frac{Q}{C_{v}}\right)^{8}\left(\frac{S}{\Delta P}\right)^{4}
$$

As can be seen from the equation there are two unknown source contribution constants $\left(C_{1}\right.$ and $\left.C_{2}\right)$. Thus, two measurement points are needed in order to find them. By comparing Equations 1 and 2, it can be seen that there are minor differences between the expressions and that the flow is still the dominating factor. This leakage model is referred to as the di-quad model and has never been published in the literature before.

\section{Data-driven Comparison Methods}

The idea behind the data-driven comparative methods that are on the right hand side of Figure 1 is to directly compare signal features of a new measurement to the signal features of other signals previously collected from leaking valves. The one that matches the best is taken to be the leak rate and hence permits a quantitative result. This method requires a large number of reference signals (a large training dataset) in order to have any value. The advantage of this method is that it does not only suggests a leak rate, but also identifies which signal has the closest overall match. This means that there is information about the type of leak if this has been documented previously as an attribute of the respective signal.

There are two companies providing AE valve leak detection and quantification in the industry today. Physical Acoustics Corporation ${ }^{\mathrm{a}}$ markets the license for the VPAC database. The VPAC database data was primarily gathered during the eighties by, among others, British Petroleum and functions as a reference dataset for leaking valves. With this data, the leak rate can be estimated on the basis of the acoustic signal level, valve type, size, and pressure. This has briefly been described by Wagner ${ }^{2}$. The other provider of AE valve leakage detection and quantification is ClampOn ${ }^{\mathrm{b}}$. Their product also uses a database for leakage rate estimation. CRANE valve services ${ }^{c}$ has a product that uses dynamic pressure sensors to determine valve leakage. The product has been described in the literature ${ }^{13}$. The leak magnitude estimation is not based on the comparative approach that the AE methods use. Instead, the DP method can only distinguish between no leakage, moderate leakage and significant leakage by monitoring the relative magnitudes in the frequency spectrum $^{29}$. There appears to be no published details on how the methods work or their performance regarding leak quantification. It is only possible to speculate on how the commercial comparative methods work and how well they work. To avoid speculation here, conservative methods will be tested against methods that can potentially produce more accurate results. Since there has not been published anything about the leak quantification methods in detail before, all the methods presented can be considered original contributions.

The instrumentation technologies mentioned are normally installed on safety critical valves by the operator as supplemental leakage monitoring. They are not considered as tools for a full functional test in the industry today. This means that it is up to the operator to decide whether or not to invest in them.

It has been shown that the leakage noise frequency spectrum changes with various factors including, the geometry of the leakage, fluid type, measurement position and leakage rate ${ }^{14}$. This indicates that the spectral distribution of the signal is of value. There are methods which use features of the probability

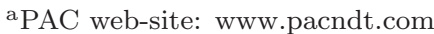

${ }^{\mathrm{b}}$ ClampOn web-site: www.clampon.com

${ }^{\mathrm{c}} \mathrm{CRANE}$ valve services web-site: www.cranevalveservices.com
} 
distribution of the signal such as variance mean and skewness. However, there are also methods that make no assumptions regarding the underlying shape of the distribution. In these methods, output coefficients (features) are generated that optimally represent the characteristics of the distributions. There are three different signal features that are utilized for the comparisons here (see Figure 1). They are:

- Signal Root Mean Square

- Frequency Spectrum Components

- ICA Mixing Vectors

(Figure 2 about here)

The frequency spectrum provides a means of characterizing the features of a signal varying in time. The raw measurement is a time series, such as those in the upper panel of Figure 2 which shows five snapshots of measurements from an AE sensor. The mean value has been removed from each trend and the variation has been scaled to unit standard deviation. Although these time trends look somehow similar to one another, it is hard to quantify the similarity. The reason why they look similar is that they contain the same range of frequencies. The frequency spectrum is the square of the magnitude of the discrete Fourier transform, and it shows what frequencies are present in the signal. For instance, the peaks in the lower panel of Figure 2 show that all five of the AE signals have similar spectra with significant frequency content at $200 \mathrm{~Hz}$ and at several other frequencies. Note that in addition to the AE frequency spectra, dynamic pressure frequency spectra are also utilized the same way in this work.

\section{Comparative Spectrum Modeling with RMS}

A signal feature that is used for direct spectrum comparison is the Root Mean Square (RMS). This feature preserves little information from the frequency spectrum of the signal. Here, the mean of the signal has not been subtracted from the signal itself before calculating the RMS. This means that the RMS is influenced by both the mean and the standard deviation, and is typically close to the mean value. The reason for presenting the RMS method in this paper is to establish a base case for comparison. The value of using spectral information would be low if the results from using the RMS of the signal are as good as or better than using the spectral features. The RMS calculation for the time varying signal produces a single value. For a new measurement this value is matched to the RMS values of the other signals by finding the minimum difference between them.

\section{Comparative Spectrum Modeling with Frequency Spectrum Components}

To fully use the information of the frequency spectrum in the direct spectrum comparison method, it is possible to use the frequency components directly derived from the Fast Fourier transform (FFT). Each row of the data matrix $(\boldsymbol{X})$ in a multivariate spectral analysis is a frequency spectrum denoted by $X(f)$, where $\boldsymbol{X}$ is the square of the magnitude of the Fourier transform and $f$ is the frequency. Since the raw measurements are sampled rather than continuous, the spectrum contains distinct frequencies $f_{i}$, known as frequency spectrum components. In this work, a sample refers to the set of data comprising the independent and dependent variable(s) from one experiment. For instance, the dependent variable would be a leakage rate and the independent variables might comprise a complete frequency spectrum with $N$ frequency channels (i.e. each sample has one dependent and $N$ independent measurements). The data matrix with distributed frequency spectrum components can be expressed as:

$N$ frequency spectrum components $\rightarrow$

$$
\boldsymbol{X}=\left(\begin{array}{ccc}
X_{1}\left(f_{1}\right) & \cdots & X_{1}\left(f_{N}\right) \\
\cdots & \cdots & \cdots \\
X_{m}\left(f_{1}\right) & \cdots & X_{m}\left(f_{N}\right)
\end{array}\right)
$$

$m$ samples (measurements) $\downarrow$ 
Valve leakage analysis using the above $\boldsymbol{X}$ matrix treats the spectrum in each row of $\boldsymbol{X}$ as a vector. Each row vector captures the spectral features of the measurement from a sensor, and spectra from the reference set are also included. The analysis proceeds by searching for similarity between vectors and thereby classifying them as belonging to one or another of the leak classes in the reference set. Suitable distance measures include the Euclidean distance in a multi-dimensional space, or the angle between the vectors. These distance measurements are described in the section about comparing feature vectors.

\section{Comparative Spectrum Modeling with Independent Components}

Principal Component Analysis (PCA) d and Independent Component Analysis (ICA) are feature extraction methods that can preserve features from the frequency spectrum. Examples of process fault detection with frequency spectrum analyses combined with PCA and ICA have been published previously ${ }^{30,31}$. The classical example these methods can solve is the cocktail party problem ${ }^{32}$. In a cocktail party, there are multiple sources of noise. The problem is to be able to separate these blind source signals by recording the noise from different positions in the room. This can be achieved with PCA or ICA by assuming that each signal recording can be expressed as a weighted sum of the noise sources. The weights depend on the distance to the noise source from the recording positions. PCA and ICA can be used to estimate the weights and noise source functions. By utilizing these methods, it is assumed that the source functions are either uncorrelated or statistically independent, respectively. Figure 3 illustrates the end result of a spectral ICA decomposition. In this case there are four spectra modeled with four independent components. The number of ICs can be reduced which makes the residual increase (the matrix $\boldsymbol{E}$ on the right hand side). On the left, the measured spectra can be seen. These have been decomposed into the independent components on the right side of the equation. Each component has a weight vector (the columns of the A matrix), whose elements indicate how influential the component is on the respective spectrum.

(Figure 3 about here)

The ICA expression can be written as:

$$
\boldsymbol{X}=\boldsymbol{A} \boldsymbol{S}^{\top}+\boldsymbol{E}
$$

Here, the $\boldsymbol{S}$ matrix is called the source matrix and the $\boldsymbol{A}$ matrix is called the mixing matrix. The difference between these components and the ones in PCA is that there is statistical independence constraint when solving for the independent components. Thus, the source matrix, $\boldsymbol{S}$, has statistically independent columns. There are several algorithms for calculating the independent components, e.g., JADE ${ }^{33}$, FastICA ${ }^{32}$, and Infomax $^{34}$.

\section{Constructing New Mixing Vectors}

It is now assumed that a mixing matrix $\left(\boldsymbol{A}_{C a l}\right)$ and a source matrix $\left(\boldsymbol{S}_{C a l}\right)$ have been calculated from a reference dataset ${ }^{\mathrm{e}}\left(\boldsymbol{X}_{C a l}\right)$. The goal now is to find the mixing vectors from the spectra of the matrix $\boldsymbol{X}_{N e w}$. New denotes spectra that are outside of the reference dataset and from, for instance, new measurements. In this paper, the spectra from the test dataset is collected in the $\boldsymbol{X}_{\text {New }}$ matrix. The vectors in the matrix are used as signatures for each of the test data spectrums. The approach taken here using ICA gives an advance on previous related work ${ }^{35}$ where PCA was used. The ICA source vectors, i.e., the columns of the source matrix, are normally used as signatures in pattern recognition. In this case, the mixing matrix is used as a signature, because the magnitude of the ICs are of more importance and not the noise source (frequency band) found in the ICs. The hypothesis is that using the mixing vectors as signal features is more robust to signal disturbances in certain frequency regions than using the frequency component vectors as signal features. The mixing vector is also normally very short compared to a frequency component vector. This

\footnotetext{
dAlso known as The Karhunen-Loève Transform (KLT), the Hotelling transform and Proper Orthogonal Decomposition (POD).

eNote that a training dataset, calibration dataset, reference dataset and a comparative dataset are the same. A model is built on the basis of these. The model is validated with test datasets.
} 
means that the information also is compressed which can, in some cases, be an advantage. Note that mean subtraction/mean centering is required prior to an PCA or ICA.

The steps of constructing a mixing vector for a new spectrum and then comparing it to the reference set can be described as follows:

1. Construct the mixing matrix and the source matrix for the reference spectrum data with an ICA analysis.

2. Use the source matrix to find the estimated mixing vector for a new spectrum.

3. Compare the new mixing vector to the reference data mixing matrix (described in the next section).

4. The best comparison match of the reference data suggests the through leakage rate and other possibly assigned attributes.

To find the mixing vector for a new spectrum, $\boldsymbol{X}_{N e w}$ is projected to the $s$ vectors:

$$
\boldsymbol{A}_{N e w}^{\top}=\left(\left(\boldsymbol{S}_{C a l}^{\top} \boldsymbol{S}_{C a l}\right)^{-1} \boldsymbol{S}_{C a l}^{\top} \boldsymbol{X}_{N e w}\right)=\boldsymbol{S}_{C a l}^{\top} \boldsymbol{X}_{N e w}
$$

When calculating one new signal at a time, the $\boldsymbol{X}_{N e w}$ and $\boldsymbol{A}_{\text {New }}$ are vectors rather than matrices and are denoted as $\boldsymbol{x}_{N e w}$ and $\boldsymbol{a}_{N e w}$. T indicates the matrix transposed. The new estimated spectrum $\left(\hat{\boldsymbol{x}}_{N e w}\right)$ based on the calculated weights and the calibrated projection vectors is:

$$
\hat{\boldsymbol{x}}_{N e w}=\boldsymbol{S}_{C a l} \boldsymbol{a}_{N e w}+\boldsymbol{e}_{N e w}
$$

The number of columns of the matrix $\boldsymbol{S}_{C a l}$ is determined by the number of ICs that have been included. The mixing vector found from the new spectrum is now the signature, or signal feature, of this particular spectrum.

\section{Comparing Feature Vectors}

The mixing vector (signature) of a new signal and the mixing vectors from the reference signals have been found. These are feature vectors that need to be compared in some way. The feature vector comparison methods suggested here are also used for the frequency component features from the other comparative spectrum method explored in this paper. Euclidean distance and angular distance are two commonly used vector comparison measurements. Both are tested in order to establish which performs the best for the purpose of through leak detection and estimation. The two measurements are presented below for comparisons between two vectors $n$ long. $X_{i}^{N e w}$ and $X_{i}^{C a l}$ are the individual vector components of a vector $X$ and where $N e w$ and $C a l$ denote a new vector and a calibration vector, respectively. In the case described in this paper, these vectors could either be the spectral frequency components or the ICA mixing vectors.

Euclidean distance:

$$
d_{e u c}=\frac{1}{n} \sqrt{\sum_{i=1}^{n}\left(X_{i}^{N e w}-X_{i}^{C a l}\right)^{2}}
$$

Angular distance:

$$
\begin{gathered}
d_{\text {ang }}=\frac{180}{\pi n} \sum_{i=1}^{n}\left(\cos ^{-1}\left(\theta_{i}\right)\right)^{2} \\
\theta_{i}=\frac{X_{i}^{\text {New }} \cdot X_{i}^{\text {Cal }}}{\operatorname{norm}\left(X_{i}^{\text {New }}\right) \cdot \operatorname{norm}\left(X_{i}^{\text {Cal }}\right)}
\end{gathered}
$$

After distances are found between the feature vectors from the reference set and the feature vector from the new measurements it is possible to rank the calibrated spectra according to their distances. This is done by finding the minimum value of the absolute distances. The method only compares which spectrum and leak rate that is the closest match to the new spectrum and the closest match indicates the leak rate. Although it is possible to create a list of all of the closest matches in the reference set, the winner will be taking it all in this case. This is more practical when there are numerous comparisons to be made for the test datasets. 


\section{Data-driven Regression Methods}

Regression is a modeling approach that attempts to model the relationship between a set of input variables (regressors), $\boldsymbol{X}$, and a set of output variables (responses), $\boldsymbol{Y}$. The variables are also often called independent and dependent variables, respectively. The general problem statement is to find a relationship of the form $\boldsymbol{Y}=\boldsymbol{X} \boldsymbol{B}+\boldsymbol{E}$, where the task is to find the regression coefficient $\boldsymbol{B}$ that minimizes the model residual $\boldsymbol{E}$. Regression can be classified as a quantitative multivariate modeling approach ${ }^{36}$. The process of finding the regression coefficients is called training or calibration. The built model can later be used for predicting response with a test set of data. Regression is typically used for modeling when there are many regressors with model impact that is uncertain. The number of variables the regression model uses (chosen by user) is the model dimensional output. These variables are called Latent Variables (LVs). There a numerous regression modeling methods that can be used (which can also been seen in Figure 1), e.g.:

- Multiple Linear Regression (MLR) ${ }^{37}$

- Principal Component Regression (PCR) ${ }^{38}$

- Independent Component Regression (ICR) ${ }^{39}$

- Partial Least Squares Regression (PLSR) ${ }^{40}$

- Kernel Partial Least Squares Regression (KPLSR) ${ }^{41}$

- Independent Component Partial Least Squares Regression (IC-PLSR) ${ }^{42}$

- Independent Component Kernel Partial Least Squares Regression (IC-KPLSR) ${ }^{43}$

- Independent Component Direct Kernel Partial Least Squares Regression (IC-DKPLSR) ${ }^{44}$

The methods that have kernel functions are able to model non-linear dependencies between the responses and regressors. Kernels are non-linear functions that can map non-linear data into a higher linear space. The regression model can then be elegantly built by solving linear algebra. There are also regression methods that can model non-linearities without the use of kernels, e.g., Neural Network PLS (NNPLS) ${ }^{45}$ and SplinePLS (SPL-PLS) ${ }^{46}$. NNPLS was not chosen for this work because KPLS is simply more robust and has a predictable performance. Spline-PLS was not used because it is similar to using polynomial kernels in KPLS, with the difference that they are based on neighboring training points (on a regular grid).

There has been little published information on how leakage rates are estimated. Wagner ${ }^{2}$ made a description of the quantification of valve leakage with $\mathrm{AE}$ instrumentation. A multiple regression analysis was conducted which resulted in a quantification model with measured AE signal, differential pressure, valve size and a valve type factor as inputs. There are no further details that can be found on the regression method itself. This paper therefore presents details on regression results for valve leakage quantification for the first time by using a selection of regression methods (see Fig. 1) with data from experiments.

The leak rates are the responses (denoted by a vector $\boldsymbol{Y}$ ) of the regression model. This enables the calculation of the leakage rate for new measurements once the regression model has been calibrated. There are two important decisions that need to be made in order to make the model. The first is which regressors to include and the second is which regression method to use. There are three different sets of regressors that will be used in the analyses. The regressors are based on:

- Conventional variables

- ICA mixing vectors

- Frequency spectrum components

The dimensions of and numerical values in the resulting $\boldsymbol{B}$ matrices are different for each type of regressors. 


\section{Conventional Variables as Regressors}

The regressors used in a leak rate estimation method that were described by Wagner ${ }^{2}$ are: measured noise signal in $\mathrm{dB}$, differential pressure, valve size and a valve type correction factor. The response variable is the leak rate. These variables can be considered to be conventional in leak rate estimation since they are currently being used in existing methods. What characterizes the conventional variables is their limited amount of signal spectral information. The purpose of using conventional variables as regressors is that they serve as a reference to the regression models that utilizes spectral information. The general regression model can be written as:

$$
\boldsymbol{Y}=\boldsymbol{X} \boldsymbol{B}+\boldsymbol{E}
$$

The rows of $\boldsymbol{Y}$ contains leak rates for each measurement in the reference datasets. While the rows of $\boldsymbol{X}$ contain the measured leak noise (the AE signal or the DP signal given in RMS) and valve differential pressure. The datasets have been categorized by the fluid type. All valve related parameters stay constant.

\section{Frequency Spectrum Components as Regressors}

The section that introduced the data-driven comparison methods described that there can be valuable information in the frequency spectrum. Each spectrum in the training dataset can be treated as a set of regressors. Each row of the $\boldsymbol{X}$ matrix in Equation 9 will therefore contain $N$ regressors if there are $N$ frequency components in each sample. Naming the frequency spectrum component matrix, $\boldsymbol{T}$, changes Equation 9 to:

$$
Y=T B+E
$$

\section{ICA Mixing Vectors as Regressors}

In the section about comparative spectrum modeling with independent components, the mixing vectors were found from an ICA analysis for each noise signal. Each mixing vector can be treated as a signature. These signature vectors can be also be used as regressors. This is similar to using spectrum frequency components because it preserves spectral information. Using the mixing vectors may yield a more robust model that can handle interfering disturbances from other acoustical sources better than using spectrum frequency components. Each row of the $\boldsymbol{X}$ matrix in Equation 9 now contains a mixing vector $(\boldsymbol{a})$ that has been found for the respective samples in the reference dataset. Equation 9 can therefore be written as:

$$
Y=A B+E
$$

There is an added stage of data compression when using this method. The first compression takes place when finding the mixing vectors in the ICA analysis. The data is compressed if the number of independent components is fewer than the number of spectra in the analysis input matrix. The second compression happens in the regression modeling when the number of latent variables is chosen.

\section{Regression Models}

The methods chosen for the regression problem here are: PLSR, KPLSR and DKPLSR. It is reasonable to choose methods that can handle linearities and non-linearities. PLSR represents the linear methods and is reported in the literature to perform as well as or better than MLR. KPLSR and DKPLSR can capture non-linearities and are therefore selected. The kernels ${ }^{\mathrm{f}}$ used in the KPLSR methods are: the polynomial kernel, the Gaussian kernel and the distance kernel.

\footnotetext{
${ }^{\mathrm{f} C o m m o n}$ kernel functions with input and output vectors $\boldsymbol{x}$ and $\boldsymbol{y}$ are: the radial basis function (Gaussian): $\kappa(\boldsymbol{x}, \boldsymbol{y})=$ $\exp \left(-\frac{\|\boldsymbol{x}-\boldsymbol{y}\|^{2}}{\sigma}\right)$, the polynomial kernel: $\kappa(\boldsymbol{x}, \boldsymbol{y})=\langle\boldsymbol{x}, \boldsymbol{y}\rangle^{r}$, the sigmoid kernel: $\kappa(\boldsymbol{x}, \boldsymbol{y})=\tanh \left(\beta_{0}\langle\boldsymbol{x}, \boldsymbol{y}\rangle+\beta_{1}\right)$, and the distance kernel: $\kappa(\boldsymbol{x}, \boldsymbol{y})=-(\text { norm }(\boldsymbol{x}-\boldsymbol{y}) / \sigma)^{r}$, where all the parameters except for $\boldsymbol{x}$ and $\boldsymbol{y}$ have to be specified and optimized. In regression, $\boldsymbol{x}$ represents the regressors and $\boldsymbol{y}$ represents the responses.
} 


\section{Model Performance Indicators}

Indicators on how well a model fits the training data and how well the model projects with the test data are needed for model evaluation. $e_{i}$ is the difference between the model response $\left(f\left(x_{i}\right)\right)$ and the measured response $\left(y_{i}\right)$.

Root Mean Square Error:

$$
\mathrm{RMSE}=\sqrt{\frac{1}{n} \sum_{i=1}^{n} e_{i}^{2}},
$$

was used both in the training process and for the testing (validation). Other performance indicators such as relative standard deviation of the error were also explored, however the results were similar.

\section{Experimental Investigations}

\section{Dataset Description}

A ball valve (442095 BSP AISI 316) with a bore diameter of $101.6 \mathrm{~mm}$ (4 in) was installed in a test rig in the Heavy Machinery Laboratory at NTNU and one of its seals was damaged to induce a through leak in the closed valve position. The leakage rate was controlled by adjusting the differential pressure across the valve and measured with a flow meter (Promass 63MT DN8, Endress-Hauser). Data was collected with an acoustical sensor (R3- $\alpha$, Physical Acoustics Corp.) mounted on the outside of the valve and a dynamic pressure sensor (M271-102, PCB Piezotronics) connected to the valve cavity. Fluids used were fresh water and nitrogen gas $\left(N_{2}\right)$. The factors that were varied within each dataset were: differential pressure across the valve (leak rate), type of leak (two types) and AE sensor position (two positions). This may leave out more minor factors such as temperature, which will have to be investigated more closely in later work. The sampling rates were two $\mathrm{MHz}$ for the acoustical sensors and $20 \mathrm{kHz}$ for the dynamic pressure sensors. Sampling time was three seconds. The frequency bands for the acoustical emission sensors were zero to 150 $\mathrm{kHz}$ and zero to $10 \mathrm{kHz}$ for the dynamic pressure sensors. The signals were transformed into the frequency spectrum by using the Fast Fourier Transform and smoothed with the Welch method ${ }^{47}$. Figure 2 showed the principle of transforming time data into frequency spectrum data. The same transformation is conducted to the time varying samples taken in the experiments. An example of some the resulting spectra in the datasets can be seen in Figure 4.

(Figure 4 about here)

The collected data have been divided into four datasets for training the models. The samples were divided according to water or gas (nitrogen) and dynamic pressure sensor or acoustic emission sensor. This means that there are in total four datasets available. Table 1 provides an overview of the number of samples in the datasets.

(Table 1 about here)

The available data were divided in to training data and validation data in order to ensure some unseen data were available to test the models. This type of validation is related to Cross-Validation $(C V)^{37}$, but without rotation of the data in the datasets. This not only is a part of the validation process of the models, but also indicates the performance of the prediction quality of the models. This is done by comparing the predicted leakages of the models and the actual leakages. For each training set there is a test dataset for model validation and performance indication purposes. Spectral signal features are derived both from AE and DP measurements. The main difference is the frequency range and the number of frequency components.

\section{Analytical and Empirical Models \\ Methods}

The models tested with the data here were the Kaewwaewnoi model (Eq. 1) and the di-quad model (Eq. 2 ). These models are only valid for liquids. Some parameters that were constant in the model's datasets were collected in master constants (e.g., $\alpha, \rho, D, C_{v}$ and $S$ were included in $C_{1}$ for Eq. 1). This makes the models dataset specific and the robustness of the models is reduced. The purpose is to uncover the potential of the models by giving them optimal constants. 
Results

The leak prediction results of the test datasets are documented in Figure 5. The Y response on the Y-axis in the upper plot represents the predicted leak rate of the model. The dimension varies with the type of fluidg used in the model data. The lower plot shows the difference between the actual response and the estimated model responses of the test data.

(Figure 5 about here)

\section{Direct Spectrum Comparison Method}

\section{Methods}

In direct spectrum comparison, features are extracted from a training dataset and compared to the features from a test dataset in order to validate the features utilized and the feature comparison method. The signal features explored were: the RMS of the signals, the ICA mixing vectors calculated from the frequency spectra, and the spectral frequency components. The feature comparison methods used were Euclidean distance and angular distance.

\section{Results}

(Figure 6 about here)

Figure 6 shows the effect of increasing the number of independent components, represented by longer mixing vectors as sample signatures. The prediction results are inaccurate when the dimensionality is approximately less than ten. This was the case for all of the tested datasets. It was enough to include 15-25 independent components, before the results coincided well with the full length spectral frequency component comparisons for all of the datasets. This means that the 25 point long signature of a mixing vector performs equally well as, in this case, a 10240 point frequency component signature. The compression is considerable. Table 2 summarizes a test on the effects of compression of the feature vectors for both the feature vector types. The frequency component signature was compressed by dividing the vectors in intervals. The number of intervals is equal to the number of the desired dimensionality (vector length). The maximum value of each interval represents the interval in the new shorter vector. The results show that both compression methods produce similar estimates when the feature vector dimension is ten and up for the Euclidean distance feature vector comparison method.

(Table 2 about here)

From Table 2 it can be seen that the Euclidean distance comparison method generally performs better than the angular distance. Angular distance seems to be unstable with some of the selected feature dimensions for this particular usage.

Using RMS as the signal feature was inferior by far compared to the spectrum feature methods with higher dimensionality as can be observed in Figure 6 and Table 2. The predicted values were better for the datasets using AE sensors than the datasets using the DP sensors (see Table 6). This indicates that the there is more useful information in the spectra of the AE measurements.

\section{Regression Methods}

Methods

Three different types of input data (signal features) were applied in the regression modeling, i.e., conventional variables, spectrum frequency components, and ICA mixing vectors. PLSR and KPLSR were chosen as regression models which represent linear and non-linear modeling respectively. Three different kernels were used in the KPLSR approach: the polynomial kernel, the Gaussian kernel and the distance kernel. The polynomial and the Gaussian kernels have one parameter to adjust each, while the distance kernel has two. Table 4 includes the resulting RMSE for one of the datasets with different types of kernels. The kernel parameters are close to optimal for each type.

\footnotetext{
${ }^{\mathrm{g}}$ In the case of the analytical models, only liquids were used as the type of fluid. But this type of plot is also used later in the paper for other modeling methods when gas is the fluid. This is why the type of plot is said to vary with the type of fluid.
} 


\section{Results}

- The distance kernel clearly performed the best for this type of data and this was also confirmed with the other datasets and the other types of regressors. Hence, the KPLSR results presented subsequently have all been using the distance kernel with close to optimal parameters.

- An important parameter to adjust in the regression models is the number of latent variables or model output dimension. The number of latent variables influences RMSE, which reduces as more latent variables are added. The decrease in RMSE achieved by increasing from 15 to 43 latent variables is minimal compared to the decrease from 5 to 15 latent variables. This trend can be observed in Table 5.

- The PLS latent variable contribution plot confirms the previous point in Figure 7. Here, most of the model is explained with approximately ten latent variables.

- The prediction results in Table 5 also demonstrate that the KPLS prediction is considerably better than the PLS prediction and that the KPLSR RMSE does not decrease rapidly with an increasing number of latent variables. The latter result implies that the KPLSR prediction is more robust with less latent variables. This trend is also observed from the other datasets, as summarized in the discussion section.

- Using ICA mixing vectors as regressors in a regression model produce different results as compared to using the frequency spectrum components as regressors directly. The effect of increasing the number of latent variables can be observed in Figure 8 where the trained model is tested with 5, 25 and 43 latent variables.

- The PLS regression model with ICA mixing vectors as inputs seemed to be unstable. The results vary considerably for every time the regression model is trained with the same data. In Table 3, the RMSE does not decrease with increasing input vector lengths, which also indicates instability. In contrast, the KPLSR models seem to increase their performance with increasing input vector lengths. Using the frequency components as model inputs consistently produce improved predictions at all compression levels compared to the ICA mixing vectors as model inputs.

The results show that the best regressors are frequency components and the number of latent variables needs to be no more than 25. KPLS regression with the distance kernel is preferred over PLSR.

(Table 3 about here)

(Table 4 about here)

(Table 5 about here)

(Figure 7 about here)

(Figure 8 about here)

\section{Comparisons and Discussions}

The results and experiences from using the experimental data with the analytical models show that they are highly sensitive to the model constants, i.e., the samples that are used to find the constants in the models. The model may fit the data to a degree with one type of failure. However, the model quickly loses the validity when another type of failure is tested with the same set constants, even though the constants should be the same in theory. This issue is illustrated for two of the models in Figure 5. The data here included two different types of leaks and the regimes of the types can easily be seen in the figure. When the models have been calibrated to one regime it translates poorly to the other regime and vice versa. The di-quad model performed in some cases better than the Kaewwaewnoi model, but this is probably due to the fact that the model is trained at two points instead of just one. The sensitivity towards the leak geometry suggests that the models will be unacceptably inaccurate if the leak geometry varies. Unfortunately in reality, it will obviously vary in many ways. This limits the use of such models to leaky valves with predictable leak geometry. The only valves with predictable leak geometry are control valves, where the valve opening is somewhat predictable. However, the objective of this work is to predict through leaks for shut-off valves. 
Using the ICA mixing vectors as signal features has proven to be an effective way of compressing data. The benefit of these compressed vectors as compared to compressed frequency components remains to be explored further in regression modeling. A procedure for compressing frequency component vectors was described in the data-driven comparison methods section for the feature vector comparison methods. The same procedure was applied to the input feature vectors for the regression models here, thus making it possible to compare the regression model results with ICA mixing vectors and spectral frequency component vectors of similar lengths as regressors. The results are summarized in Table 3 for one of the datasets. The number of latent variables is the same as the input vector lengths, with the exemption of the full length input vector for the frequency components where the regression model output dimension is 54 .

(Table 6 about here)

Table 6 summarizes the prediction results from all of the regression models in RMSE for all of the datasets. The datasets were divided into two liquid and two gas datasets. For each of these fluids, one dataset was made for the AE sensor and the other for the DP sensor. From the table it can be observed that:

- The regression models based on the AE datasets produce more accurate leak predictions than the DP datasets.

- The model input feature type with the most accuracy was the spectral frequency components, closely followed by ICA mixing vector features. These spectral features scored much better than using the conventional variables as model inputs.

- The non-linear modeling method KPLSR, has achieved improved prediction results in all situations, compared to its linear modeling counterpart, PLS.

- The liquid datasets gave slightly better predictions than the gas datasets for all the sensors.

The difference between the algorithms in DKPLSR and ordinary KPLSR were some negligible differences in the regression model training residual, none of which had any impact on the prediction results.

The model will be robust against changes in the measurement position when there is a large variation of the sensor positioning when the training set for the model is built. This is therefore a factor that needs to be varied as much as possible when collecting a reference database. Using multiple sensors at the same time is possible in this case and it will also make the model more robust against, e.g., different valve designs.

The experimental results and analyses suggest that the best way forward is to start collecting a comprehensive database of acoustical and dynamic pressure data from leaking reference valves with an emphasis on recording the frequency spectra. One for gas and one for liquid would be needed. The results have shown that the leak rate estimation can be improved by utilizing the methods suggested in this paper. Improved leakage rate estimation results in safer operations and reduced maintenance costs. Interested parties would be from industry that focuses on high standards within process safety and that have access to leaking valves in need of maintenance, i.e., the chemical process industry (The VPAC database mentioned in the datadriven comparison methods section was financed by operators from the oil \& gas industry.) and the nuclear power plant industry. The latter suggested industry would also be concerned with steam as the fluid of interest.

\section{Conclusion}

Modeling an internal leak in a valve with unknown leak geometry is inherently difficult. Models based on analytical relationships can be reasonable if the geometry is predictable. However, in practise, they are unknown. The work shows that current methods for leak estimation can be improved. Information from acoustic emission sensors and dynamic pressure sensors is of great value, and the results suggest these should be fitted routinely to safety critical shut-down valves. For predicting through leaks in shut-down valves, it is reasonable to use a comparative approach, i.e., to compare a leaky valve with a group of other leaky valves with known leakage rates. In this paper, comparative approaches have been explored based on 
sensor signals from dynamic pressure sensors and acoustical emission sensors that allow for spectral data. A direct comparison approach with spectral frequency components as signal features is recommended for the identification of leakage types. For leakage rate quantification, the non-linear regression method, KPLSR, with the distance kernel, performed the best. Current methods for leak estimation can be improved by exploiting the results from this paper by either modifying the current comparative approaches being used, or by building a new database of leaking reference valves. If the leakage rate of a valve can be monitored more accurately, it will lead to improvements to the planning of maintenance actions, which ultimately cuts costs, by, e.g., delaying the replacement of a safety critical valve, or having less functional test, which reduces system downtime. Operations will also be safer because of the improved condition monitoring.

\section{References}

[1] Kaewwaewnoi W, Prateepasen A, Kaewtrakulpong P. Investigation of the relationship between internal fluid leakage through a valve and the acoustic emission generated from the leakage Measurement. 2010;43:274-282.

[2] Wagner H. Innovative techniques to deal with leaking valves Technical Papers of ISA. 2004;454:105-117.

[3] Dickey J, Dimmick J, Moore PM. Acoustic Measurement of Valve Leakage Rates Materials Evaluation. 1978;36:67-77.

[4] Dimmick JG, Nicholas JR, Dickey JW, Moore PM. Acoustical Valve Leak Detector For Fluid System Maintenance Naval Engineers Journal. 1979;91:71-83.

[5] El-Shorbagy KA. An investigation into noise radiation from flow control valves with particular reference to flow rate measurement Applied Acoustics. 1983;16:169-181.

[6] Lee JH, Lee MR, Kim JT, Luk V, Jung YH. A study of the characteristics of the acoustic emission signals for condition monitoring of check valves in nuclear power plants Nuclear engineering and design. 2006;236:1411-1421.

[7] Lee SG, Park JH, Yoo KB, Lee SK, Hong SY. Evaluation of internal leak in valve using acoustic emission method Key Engineering Materials. 2006;326-328 I:661-664.

[8] Pollock AA, Hsu SYS. Leak detection using acoustic emission Journal of Acoustic Emission. 1982;1:237-243.

[9] Shack WJ, Ellingson WA, Youngdahl CA. Development of a Noninvasive Acoustic Leak Detection System for Large High Pressure Gas Valves ISA Transactions. 1980;19:65-71.

[10] Sharif MA, Grosvenor RI. Internal valve leakage detection using an acoustic emission measurement system Transactions of the Institute of Measurement and Control. 1998;20:233-242.

[11] Thompson G, Askari AR. Air Leak Detection Through Ball Plug Valves by Vibration Monitoring Int. Conf. on Dev. in Valves and Actuators for Fluid Control. 1985:319-327.

[12] Thompson G, Zolkiewski G. Experimental investigation into the detection of internal leakage of gases through valves by vibration analysis Proceedings of the Institution of Mechanical Engineers, Part E: Journal of Process Mechanical Engineering. 1997;211:195-207.

[13] Juvik T, Hermansen T, Carr R, Hale S. Online valve monitoring systems used on off-shore platforms in the north sea in 21st International Conference on Offshore Mechanics and Artic Engineering(Oslo, Norway):5 2002.

[14] Meland E, Henriksen V, Hennie E, Rasmussen M. Spectral Analysis of Internally Leaking Shut-Down Valves Accepted to Journal of Measurement. 2011.

[15] Allen EE. Control Valve Noise In ISA handbook of control valves. 1976:221-244.

[16] Baumann HD, Kiesbauer J. A method to estimate hydrodynamic noise produced in valves by submerged turbulent and cavitating water jets Noise Control Eng.. 2004;52:49-55.

[17] Izmit A, McDaniel OH, Reethof G. The nature of noise sources in control valves Proc. Inter-Noise 77, Zurich, Switzerland. 1977:B183-B188.

[18] Jenvey PL. Gas pressure reducing valve noise Journal of Sound and Vibration. 1975;41:506-509.

[19] Reethof G. Turbulence-Generated Noise in Pipe Flow Ann. Rev. Fluid Mech.. 1978;10:333-367.

[20] Reethof G, Karvelis AV. Internal wall pressure field studies downstream from orificial-type valves ISA/74 Conf. and Ex., NY. $1974 ; 78$

[21] Robertson JE. Fluid dynamic noise generation by control valves Proc. Noise, Expo.73. 1973:180-185.

[22] Sawley RJ, White PH. The influence of pressure recovery on the development of gas vavle noise descriptions $I S A A C$ Advances in Instrumentation. 1974;29:9.

[23] Schuder CB. New technique for valve noise prediction Chemical Processing, Nov. 10-15. 1970:10-15.

[24] Small DJ. The noise of gas regulator valves The Inst. of Gas Eng., 38th Autumn Research Meeting, London. $1972: 20$.

[25] Small DJ, Davies POAL. A computerized valve noise predictions system Noise Control Engineering. 1975;4:124-128.

[26] Zarate RA. Control Valve Noise Prediction and Sizing ISA AC Advances in Instrumentation. 1974;29:6.

[27] Lyons JL. Lyons' Encyclopedia of Valves. Krieger Publishing Companyreprint 1993 ed. 1975.

[28] Curle N. The influence of solid boundaries upon aerodynamic sound Proc. Royal Society. 1955;A232:505.

[29] Heagerty D, Leon R. Method and Apparatus for On-line Detection of Leaky Emergency Shutdown Valves World intellectual property organization, PCT/US98/12868. 1999:45.

[30] Thornhill NF, Shah SL, Huang B, Vishnubhotla A. Spectral principle component analysis of dynamic process data Control Engineering Practice. 2002;10:883-846.

[31] Xia C, Howell J, Thornhill NF. Detecting and isolating multiple plant-wide oscillations via spectral independent component analysis Automatica. 2005;41:2067-2075. 
[32] Hyvärinen A, Oja E. Independent Component Analysis: Algorithms and Applications Neural Networks. 2000;13:411-430.

[33] De Lathauwer LD, De Moor B, Vanderwalle J. An introduction to independent component analysis Journal of Chemometrics. 2000;14:123-149.

[34] Cardoso J, Westad F. Blind beamforming for non-Gaussian signals IEE Proc.-F. 1993;140:362-370.

[35] Tan CC, Thornhill NF, Belchamber RM. Principal component analysis of spectra with application to acoustic emissions from mechanical equipment Trans. of the Inst. of Measurement and Control. 2002;24:333-353.

[36] Venkatasubramanian V, Rengaswamy R, Yin K, Kavuri SN. A review of process fault detection and diagnosis Part I: Quantitative model-based methods Computers and Chemical Eng.. 2003;27:293-311.

[37] Wold S, Sjöström M, Eriksson L. PLS-regression: a basic tool of chemometrics Chemometrics and Intelligent Laboratory Systems. 2001;58:109-130.

38] Mardia K, Kent J, Bibby J. Multivariate Analysis. Academic Press, London1st ed. 1980.

[39] Chen J, Wang XZ. A new approach to Near-Infrared Spectral Data Analysis using Independent Component Analysis J. Chem. Inf. Comput. Sci. 2001;41:992-1001.

[40] Geladi P, Kowalski BR. Partial Least-Squares Regression: A Tutorial Analytica Chimica Acta. 1986;185:1-17.

[41] Rosipal R, Trejo LJ. Kernel Partial Least Squares Regression in Reproducing Kernel Hilbert Space Journal of Machine Learning Research. 2001;2:97-123.

[42] Westad F. Independent component analysis and regression applied on sensory data J. Chemometrics. 2005;19:171-179.

[43] Zhang Y, Zhang Y. Complex process monitoring using modified partial least squares method of independent component regression Chemometrics and Intelligent Laboratory Systems. 2009;98:143-148.

[44] Bennett KP, Embrechts MJ. An optimization perspective on Kernel Partial Least Squares Regression Advances in Learning Theory: Methods, Models and Applications, NATO Science Series III: Computer \& Systems Sciences. 2003;190:227-250.

[45] Baffi G, Martin EB, Morris AJ. Nonlinear projection to latent structures revisited (the neural network PLS algorithm) Computers \& Chemical Engineering. 1999;23:1293-1307.

[46] Wold S. Nonlinear partial least squares modeling II. Spline inner relation Chemometrics and Intelligent Laboratory Systems. 1992;14:71-84.

[47] Welch PD. The use of fast Fourier transforms for the estimation of power spectra: A method based on time averaging over short, modified periodograms IEEE Trans. on Audio and Electroacoustics. 1967;AU-15:17-20. 


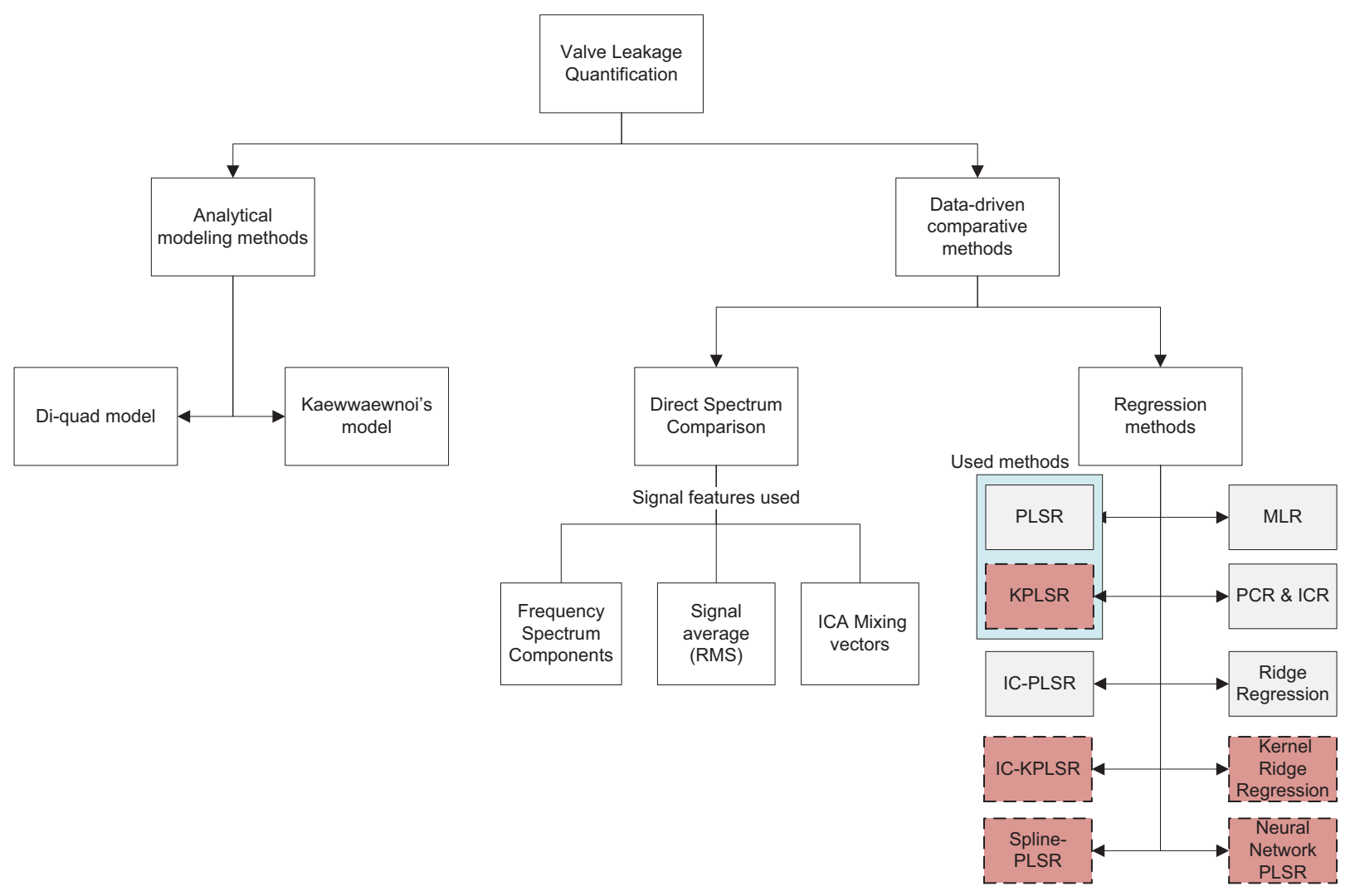

Figure 1: An overview of potential leak model candidates explored. The red squares indicate non-linear methods. 

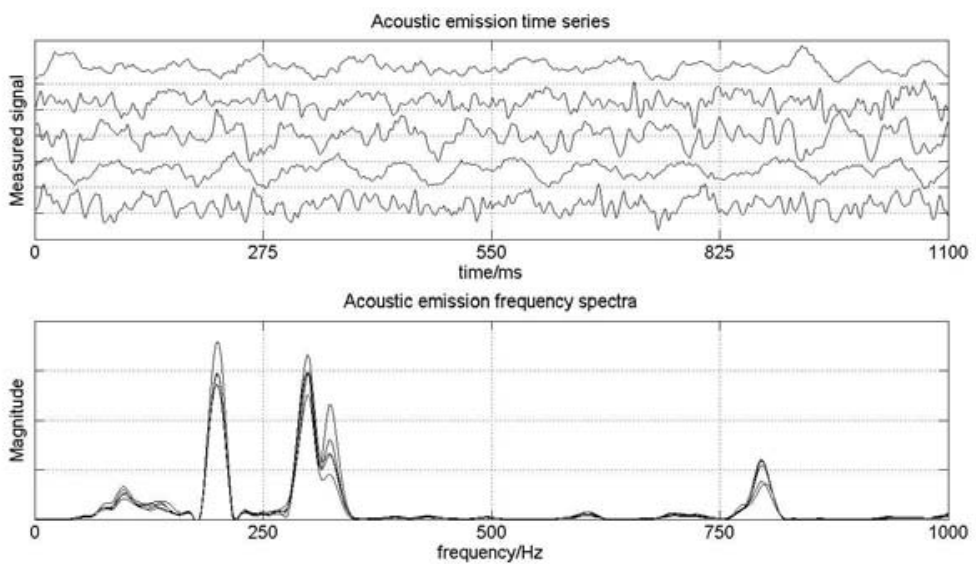

Figure 2: Time series can be transformed to frequency spectra with a Fast Fourier Transformation. The frequency spectra indicate at which frequencies the time series are oscillating. This example shows five time series (upper panel) with similar frequency spectra (lower panel). 


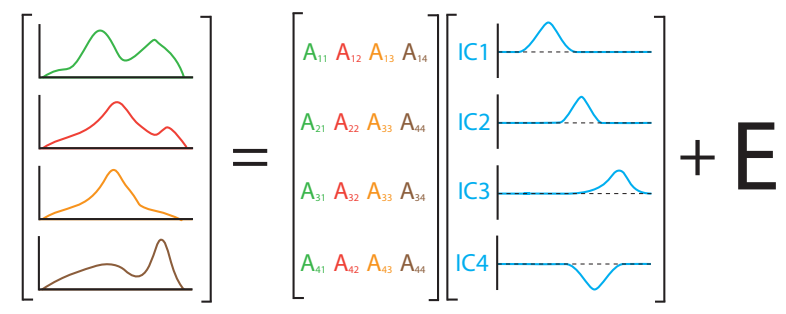

Figure 3: The Principle Behind ICA Spectral Decomposition 


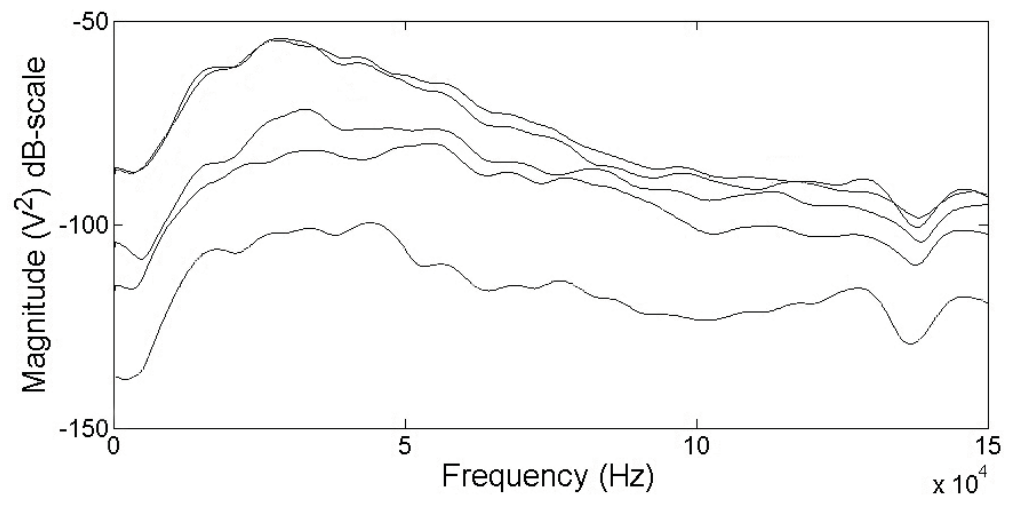

Figure 4: The frequency spectra are obtained after the measured time series have been sampled. The curves are examples of AE samples taken with different differential pressures (leak rates) across the valve. 

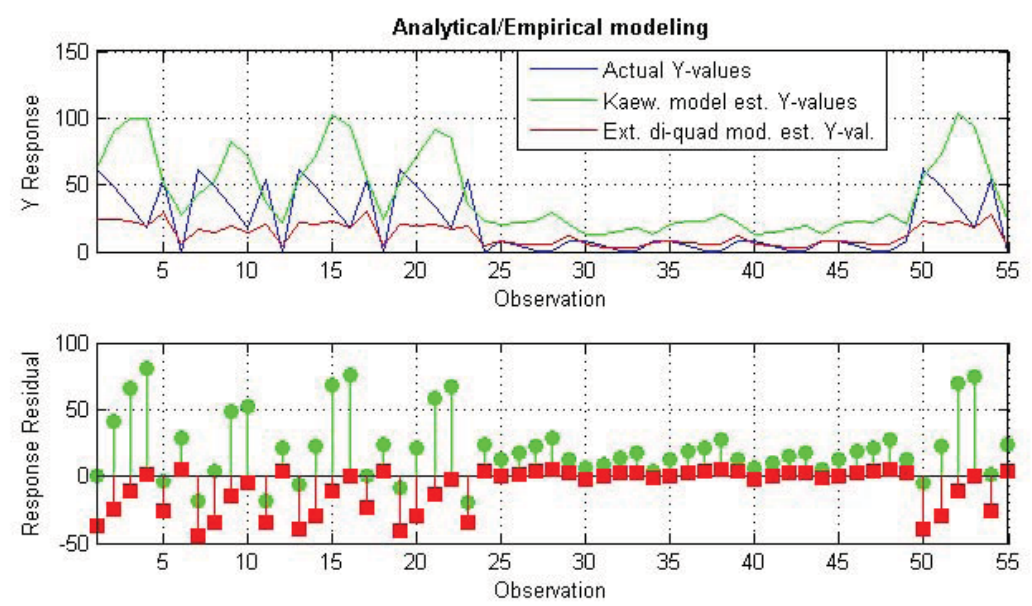

Figure 5: The analytical models are highly dependent on the constants found for their models. Any change to the type of leak can impact the model negatively. The test data here contains two leak type regimes which can be distinguished in the plots. 

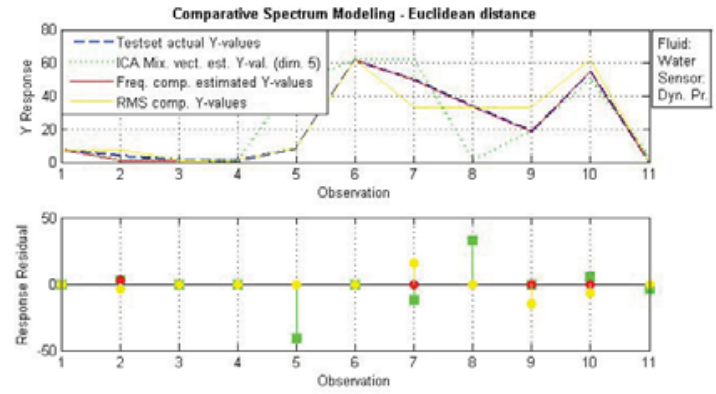

(a) ICA mixing Vector dimension: 5
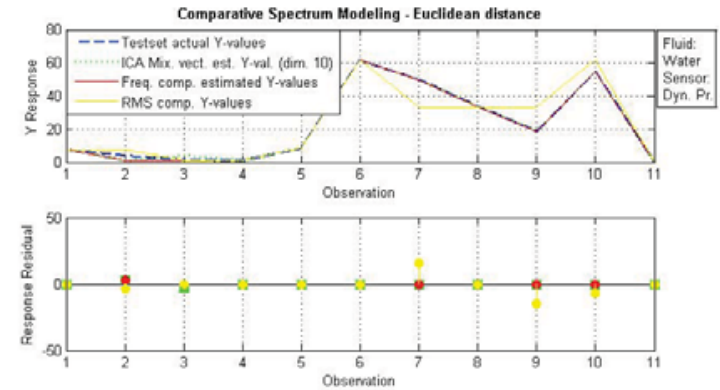

(b) ICA mixing Vector dimension: 10

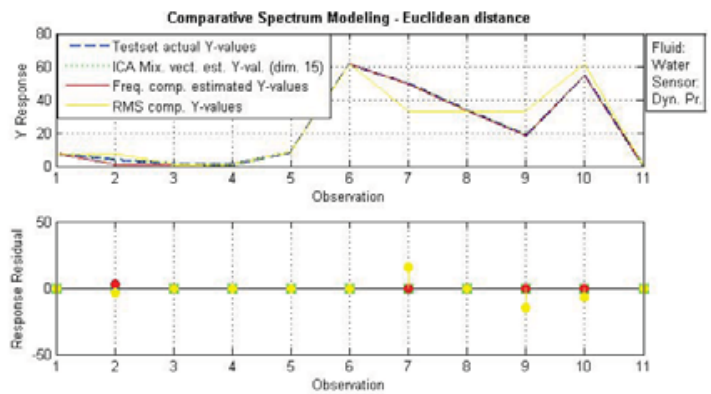

(c) ICA mixing Vector dimension: 15

Figure 6: Increasing the dimensions in the Spectral Comparison Model 


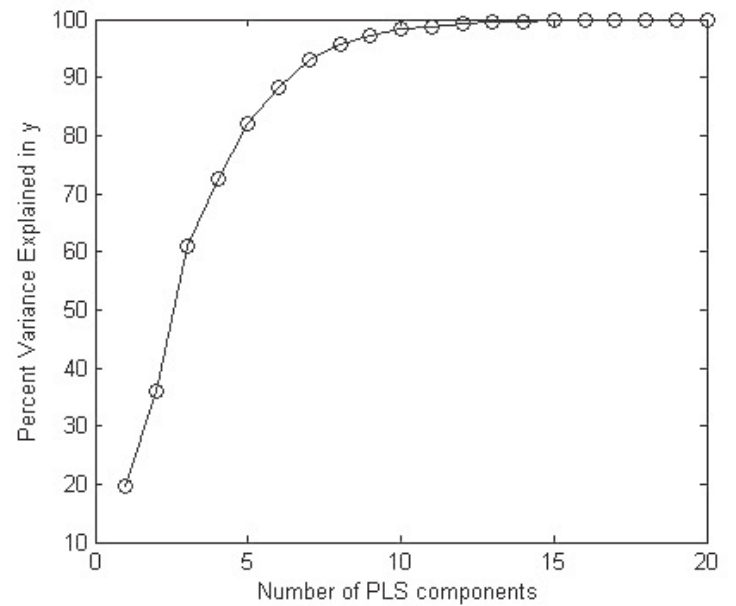

Figure 7: The contribution of the latent variables for the response in a PLS regression model. 

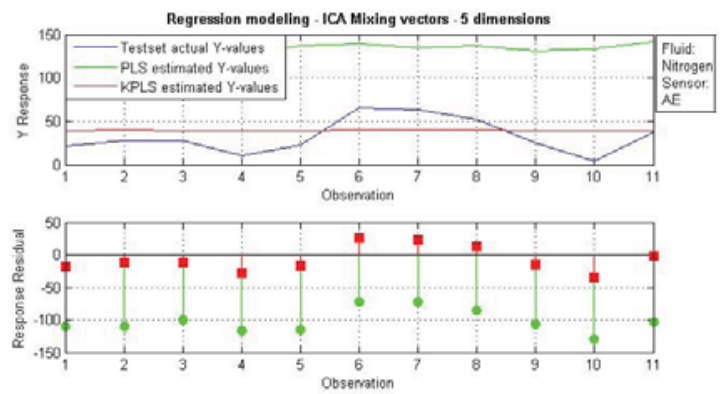

(a) Mixing vector dimension: 5
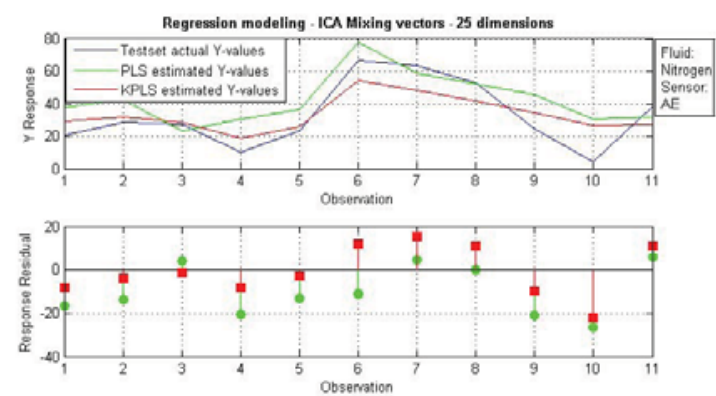

(b) Mixing vector dimension: 25
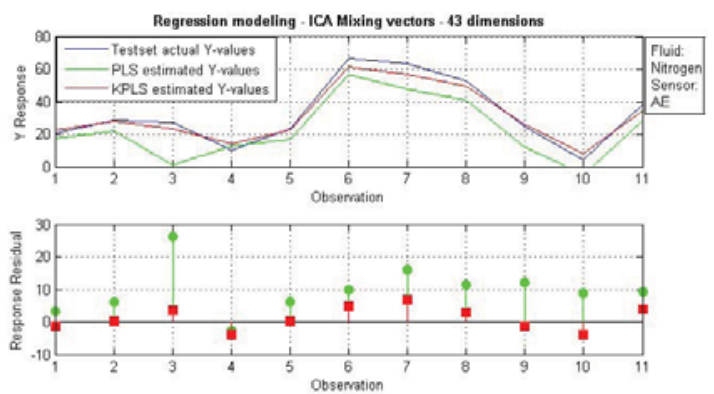

(c) Mixing vector dimension: 43

Figure 8: Increasing the dimensions of the regression models with ICA mixing vectors as regressors. The number of latent variables is the same as the mixing vector dimension. The plots clearly show that the model prediction is very inaccurate at a low number of latent variables. The model increases accuracy with an increasing number of latent variables because the length of the input mixing vectors is determined by the number of latent variables. Reducing the number of latent variables will also reduce the information (vector length) in the model input vectors. 
Table 1: An overview of the number of samples in the reference and test datasets.

\begin{tabular}{|c|c|c|c|c|}
\hline $\begin{array}{c}\text { Dataset } \\
\text { number }\end{array}$ & Fluid & Sensor & $\begin{array}{c}\text { Number of samples } \\
\text { in reference set }\end{array}$ & $\begin{array}{c}\text { Number of samples } \\
\text { in test set }\end{array}$ \\
\hline 1 & Water & AE & 44 & 11 \\
2 & Water & DP & 55 & 11 \\
3 & Nitrogen & AE & 44 & 11 \\
4 & Nitrogen & DP & 55 & 11 \\
\hline
\end{tabular}


Table 2: Increasing ICA mixing vectors and frequency component feature dimensions. These features should score lower than the RMS comparison in order for them to be viable as signal features in the comparison approach. The fluid is water and the type of sensor is dynamic pressure in this dataset. It is possible for the RMSE to be zero because the samples in the training set do not have unique leak rates.

\begin{tabular}{|c|c|c|}
\hline $\begin{array}{c}\text { Comparison } \\
\text { basis }\end{array}$ & $\begin{array}{c}\text { RMSE } \\
\text { (Euc. dist.) }\end{array}$ & $\begin{array}{c}\text { RMSE } \\
\text { (Ang. dist.) }\end{array}$ \\
\hline RMS comp. & 7.0 & N/A \\
\hline Freq. comp. & 5.3 & 17.0 \\
dim. 5 & 0 & 3.7 \\
dim. 10 & 0 & 10.9 \\
dim. 15 & 0 & 10.9 \\
dim. 20 & 0.9 & 10.9 \\
dim. 25 & 0.9 & 0 \\
Full length & & \\
\hline ICA Mix. & 25.8 & 16.0 \\
dim. 5 & 3.6 & 0.9 \\
dim. 10 & 4.6 & 0.9 \\
dim. 15 & 0.8 & 0.8 \\
dim. 20 & 0 & 0 \\
dim. 25 & 0 & 0.8 \\
dim. 55(max) & 0
\end{tabular}


Table 3: Comparing the compression impact of the two types of signal features in the regression modeling for one of the datasets.

\begin{tabular}{|c|cc|cc|}
\hline & \multicolumn{2}{|c|}{ ICA Mixing Vectors } & \multicolumn{2}{c|}{ Frequency Components } \\
\hline $\begin{array}{c}\text { Input } \\
\text { vector lengths }\end{array}$ & $\begin{array}{c}\text { PLSR test } \\
\text { accuracy RMSE }\end{array}$ & $\begin{array}{c}\text { KPLSR test } \\
\text { accuracy RMSE }\end{array}$ & $\begin{array}{c}\text { PLSR test } \\
\text { accuracy RMSE }\end{array}$ & $\begin{array}{c}\text { KPLSR test } \\
\text { accuracy RMSE }\end{array}$ \\
\hline Full length & N/A & N/A & 16.6 & 4.7 \\
54 & 18.2 & 6.2 & 103.8 & 5.2 \\
30 & 10.1 & 8.0 & 13.9 & 5.5 \\
20 & 31.5 & 7.2 & 11.4 & 6.4 \\
10 & 15.0 & 9.2 & 12.2 & 7.0 \\
5 & 23.8 & 27.0 & 15.6 & 10.5 \\
\hline
\end{tabular}


Table 4: Comparing the performance of three different kernels. Regression output dimension is 25. Fluid is Nitrogen and sensor type is AE. The parameters for each kernel are close to optimal.

\begin{tabular}{|c|c|c|}
\hline \multirow[b]{2}{*}{$\begin{array}{c}\text { Regression } \\
\text { Method }\end{array}$} & \multicolumn{2}{|c|}{ RMSE } \\
\hline & $\begin{array}{l}\text { Training } \\
\text { accuracy }\end{array}$ & $\begin{array}{c}\text { Test } \\
\text { accuracy }\end{array}$ \\
\hline PLSR - N/A & 0.06 & 6.65 \\
\hline KPLSR - Polynomial kernel & 0.15 & 5.78 \\
\hline KPLSR - Gaussian kernel & 0.02 & 5.12 \\
\hline KPLSR - Distance kernel & $7 \mathrm{e}-6$ & 3.21 \\
\hline
\end{tabular}


Table 5: This table shows that using all the possible latent variables is not necessary. The test accuracy does not decrease considerably with an increasing number of latent variables. The reference dataset here is for the AE sensor with nitrogen as the fluid. The frequency spectrum components are used as regressors and the kernel parameters used are optimal.

\begin{tabular}{|c|c|c|}
\hline $\begin{array}{c}\text { Latent } \\
\text { Variables }\end{array}$ & $\begin{array}{c}\text { KPLS Test } \\
\text { Accuracy (RMSE) }\end{array}$ & $\begin{array}{c}\text { PLS Test } \\
\text { Accuracy (RMSE) }\end{array}$ \\
\hline 5 & 3.6 & 10.5 \\
15 & 3.2 & 7.1 \\
43 & 3.2 & 6.7 \\
\hline
\end{tabular}


Table 6: Regression model prediction summary for all of the datasets in RMSE. Maximum output dimensionality for all the regression methods is applied and close to optimal distance kernels are used.

\begin{tabular}{|c|c|r|r|r|r|r|}
\hline $\begin{array}{c}\text { Regression } \\
\text { input }\end{array}$ & $\begin{array}{c}\text { Test } \\
\text { performance }\end{array}$ & $\begin{array}{r}\text { Dataset } \\
\text { Water AE }\end{array}$ & $\begin{array}{r}\text { Dataset } \\
\text { Water DP }\end{array}$ & $\begin{array}{c}\text { Dataset } \\
\text { Gas AE }\end{array}$ & $\begin{array}{r}\text { Dataset } \\
\text { Gas DP }\end{array}$ & $\begin{array}{r}\text { Method } \\
\text { average }\end{array}$ \\
\hline Frequency comp. & PLS - RMSE & 2.9 & 16.6 & 6.7 & 24.0 & 12.5 \\
Frequency comp. & KPLS - RMSE & 0.9 & 4.7 & 3.2 & 4.5 & 3.3 \\
\hline ICA mix. comp. & PLS - RMSE & 11.4 & 18.2 & 12.0 & 29.8 & 17.8 \\
ICA mix. comp. & KPLS - RMSE & 0.8 & 6.2 & 3.6 & 6.7 & 4.3 \\
\hline Conventional var. & PLS - RMSE & 17.4 & 15.9 & 15.6 & 19.1 & 17.0 \\
Conventional var. & KPLS - RMSE & 12.9 & 11.9 & 9.9 & 9.6 & 11.1 \\
\hline
\end{tabular}

